Proc. of the Fourth International Conference on Advances in Computing, Electronics and Communication - ACEC 2016. Copyright (C) Institute of Research Engineers and Doctors. All rights reserved. ISBN: 978-1-63248-113-9 doi: 10.15224/ 978-1-63248-113-9-07

\title{
A New Note on Summability of Factored Infinite
}

\section{Series}

Şebnem YILDIZ

Abstract-In this paper, two main theorem dealing with infinite and factored Fourier series, which generalizes some known results, has been generalized to $\left|A, p_{n} ; \delta\right|_{k}$ summability method. This new theorem also includes several known and new results.

Keywords- Summability factors, absolute matrix summability, Fourier series, infinite series, Hölder inequality, Minkowski inequality.

\section{Introduction}

Definition 1. Let $\sum a_{n}$ be a given infinite series with partial sums $\left(s_{n}\right)$. Let $\left(p_{n}\right)$ be a sequence of positive numbers such that

$$
P_{n}=\sum_{v=0}^{n} p_{v} \rightarrow \infty, n \rightarrow \infty,\left(P_{-i}=p_{-i}=0, i \geq 1\right)
$$

The sequence-to-sequence transformation

$$
\sigma_{n}=\frac{1}{P_{n}} \sum_{v=0}^{n} p_{v} s_{v}
$$

defines the sequence $\left(t_{n}\right)$ of the Riesz mean or simply the $\left(\bar{N}, p_{n}\right)$ mean of the sequence $\left(s_{n}\right)$ generated by the sequence of coefficients $\left(p_{n}\right)$ (see [11]).

Definition 2. The series $\sum a_{n}$ is said to be summable $\left|\bar{N}, p_{n}\right|_{k}, k \geq 1$ if (see [3])

$$
\sum_{n=1}^{\infty}\left(\frac{P_{n}}{p_{n}}\right)^{k-1}\left|\Delta \sigma_{n-1}\right|^{k}<\infty .
$$

In the special case when $p_{n}=1$ for all values of $\mathrm{n}$ (resp. $k=1$ ), $\left|\bar{N}, p_{n}\right|_{k}$ summability is the same as $|C, 1|_{k}\left(\right.$ resp. $\left.\left|\bar{N}, p_{n}\right|\right)$ summability (see [10]).

Şebnem YILDIZ

Ahi Evran University

Turkey
Definition 3. The series $\sum a_{n}$ is said to be summable $\left|\bar{N}, p_{n} ; \delta\right|_{k}, k \geq 1$ and $\delta \geq 0$ if (see [7])

$$
\sum_{n=1}^{\infty}\left(\frac{P_{n}}{p_{n}}\right)^{\delta k+k-1}\left|\Delta \sigma_{n-1}\right|^{k}<\infty .
$$

where $\Delta \sigma_{n-1}=-\frac{p_{n}}{P_{n} P_{n-1}} \sum_{v=1}^{n} P_{v-1} a_{v}, \quad n \geq 1$.

In the special case, when $p_{n}=1$ for all values of $\mathrm{n}$ (resp. $\delta=0),\left|\bar{N}, p_{n} ; \delta\right|_{k}$ summability is the same as $|C, 1 ; \delta|_{k}\left(\right.$ resp. $\left.\left|\bar{N}, p_{n}\right|_{k}\right)$ summability.

\section{Known Results}

Many works dealing with some absolute summability methods of infinite and Fourier series have been done (see [1-2], [4-8], [12-15],[21]). Among them, in [16] Özarslan has proved the following theorem.

Theorem 1. Let $k \geq 1$. If the sequence $\left(s_{n}\right)$ is bounded and the sequences $\left(\lambda_{n}\right)$ and $\left(p_{n}\right)$ satisfy the following conditions

$$
\begin{gathered}
\sum_{n=1}^{m} p_{n}\left|\lambda_{n}\right|=O(1) \quad \text { as } n \rightarrow \infty, \\
\sum_{n=1}^{m} p_{n}\left|\Delta \lambda_{n}\right|=O(1) \quad \text { as } n \rightarrow \infty, \\
p_{n+1}=O\left(p_{n}\right),
\end{gathered}
$$

then the series $\sum a_{n} \lambda_{n} P_{n}$ is summable $\left|\bar{N}, p_{n}\right|_{k}, k \geq 1$.

\section{An Application of Absolute Matrix Summability to Fourier Series}

Let $f(x)$ be a periodic function with period $2 \pi$ and Lebesgue integrable over $(-\pi, \pi)$. The Fourier series of $f(x)$ is 
Proc. of the Fourth International Conference on Advances in Computing, Electronics and Communication - ACEC 2016. Copyright (c) Institute of Research Engineers and Doctors. All rights reserved.

ISBN: 978-1-63248-113-9 doi: 10.15224/ 978-1-63248-113-9-07

$$
f(x) \square \frac{1}{2} a_{0}+\sum_{n=1}^{\infty}\left(a_{n} \cos n x+b_{n} \sin n x\right)=\sum_{n=0}^{\infty} C_{n}(x)
$$

where $\left(a_{n}\right)$ and $\left(b_{n}\right)$ denote the Fourier coefficients.

The convergence of Fourier series can be ensured by local hypothesis, that is to say, the behavior of the convergence of Fourier series for a particular value of $x$ depends on the behavior of the function in the immediate neighbourhood of this point only (see [20]).

Theorem 2. ([16]) Let $k \geq 1$. The summability $\left|\bar{N}, p_{n}\right|_{k}$ of the series $\sum C_{n}(x) \lambda_{n} P_{n}$ at a point is a local property of a generating function if the conditions (5) and (6) are satisfied.

Definition 4. Let $\sum a_{n}$ be a given infinite series with the partial sums $\left(s_{n}\right)$. Let $A=\left(a_{n v}\right)$ be a normal matrix, i.e., a lower triangular matrix of nonzero diagonal entries. Then A defines the sequence-to-sequence transformation, mapping the sequence $s=\left(s_{n}\right)$ to $A s=\left(A_{n}(s)\right)$, where

$$
A_{n}(s)=\sum_{v=0}^{n} a_{n v} s_{v}, \quad n=0,1, \ldots
$$

The series $\sum a_{n}$ is said to be summable $|A|_{k}, k \geq 1$ if (see [19])

$$
\sum_{n=1}^{\infty} n^{k-1}\left|\bar{\Delta} A_{n}(s)\right|^{k}<\infty
$$

and it is said to be summable $\left|A, p_{n}\right|_{k}, k \geq 1$, if (see [18])

$$
\sum_{n=1}^{\infty}\left(\frac{P_{n}}{p_{n}}\right)^{k-1}\left|\bar{\Delta} A_{n}(s)\right|^{k}<\infty
$$

where

$$
\bar{\Delta} A_{n}(s)=A_{n}(s)-A_{n-1}(s) .
$$

If we take $p_{n}=1$ for all values of $\mathrm{n},\left|A, p_{n}\right|_{k}$ summability is the same as $|A|_{k}$ summability. Also, if we take $a_{n v}=\frac{p_{v}}{P_{n}}$, then $\left|A, p_{n}\right|_{k}$ summability is the same as $\left|\bar{N}, p_{n}\right|_{k}$ summability.

and also it is said to be summable $\left|A, p_{n} ; \delta\right|_{k}, k \geq 1$, and $\delta \geq 0$ if (see [17])

$$
\sum_{n=1}^{\infty}\left(\frac{P_{n}}{p_{n}}\right)^{\delta k+k-1}\left|\bar{\Delta} A_{n}(s)\right|^{k}<\infty
$$

\section{Main Results}

The aim of this paper is to prove a more general theorem which includes some of the above-mentioned result as a special cases.

Theorem 3. Let $k \geq 1$ and $0 \leq \delta<1 / k$. Let $\left(s_{n}\right)$ be a bounded sequence and suppose that $A=\left(a_{n v}\right)$ is a positive normal matrix such that

$$
\begin{gathered}
\bar{a}_{n 0}=1, \quad n=0,1, \ldots, \\
a_{n-1, v} \geq a_{n v} \text { for } 1 \leq v \leq n-1, \\
a_{n n}=O\left(\frac{p_{n}}{P_{n}}\right),
\end{gathered}
$$

$\sum_{n=v+1}^{m+1}\left(\frac{P_{n}}{p_{n}}\right)^{\delta k}\left|\Delta_{v}\left(\hat{a}_{n v}\right)\right|=O\left\{\left(\frac{P_{v}}{p_{v}}\right)^{\delta k-1}\right\}$ as $m \rightarrow \infty$, $\sum_{n=v+1}^{m+1}\left(\frac{P_{n}}{p_{n}}\right)^{\delta k}\left|\hat{a}_{n, v+1}\right|=O\left\{\left(\frac{P_{v}}{p_{v}}\right)^{\delta k}\right\}$ as $m \rightarrow \infty$.

If a sequence $\left(\lambda_{n}\right)$ and $\left(p_{n}\right)$ holds the following conditions,

$$
\begin{aligned}
& \sum_{n=1}^{m}\left(\frac{P_{n}}{p_{n}}\right)^{\delta k} p_{n}\left|\lambda_{n}\right|=O(1) \text { as } \quad m \rightarrow \infty, \\
& \sum_{n=1}^{m}\left(\frac{P_{n}}{p_{n}}\right)^{\delta k} p_{n}\left|\Delta \lambda_{n}\right|=O(1) \text { as } \quad m \rightarrow \infty,
\end{aligned}
$$$$
p_{n+1}=O\left(p_{n}\right)
$$

then the series $\sum a_{n} \lambda_{n} P_{n}$ is summable $\left|A, p_{n} ; \delta\right|_{k}$.

Theorem 4. Let $k \geq 1$ and $0 \leq \delta<1 / k$. The summability $\left|A, p_{n} ; \delta\right|_{k}$ of the series $\sum C_{n}(x) \lambda_{n} P_{n}$ at a point is a local property of a generating function if all the conditions of Theorem 3 are satisfied.

We need the following lemma for the proof of our theorem. Lemma 5 (see [16]) If the sequences $\left(\lambda_{n}\right)$ and $\left(p_{n}\right)$ satisfy the conditions (5) and (6) of Theorem 1, then $P_{m}\left|\lambda_{m}\right|=O(1)$ as $m \rightarrow \infty$. 


\section{Proof of Theorem 3}

$\left(I_{n}\right)$ denotes the A-transform of the series $\sum a_{n} \lambda_{n} P_{n}$. Then, we have

$$
\bar{\Delta}_{n}=\sum_{v=1}^{n} \hat{a}_{n v} a_{v} \lambda_{v} P_{v} .
$$

Applying Abel's transformation to this sum, we get that

$$
\begin{aligned}
\bar{\Delta} I_{n}(x) & =\sum_{v=1}^{n-1} \Delta_{v}\left(\hat{a}_{n v} \lambda_{v}\right) P_{v} \sum_{r=1}^{v} a_{r}+\hat{a}_{n n} \lambda_{n} P_{n} \sum_{v=1}^{n} a_{v} \\
& =\sum_{v=1}^{n-1} \Delta_{v}\left(\hat{a}_{n v} \lambda_{v} P_{v}\right)+a_{n n} \lambda_{n} P_{n} s_{n} \\
& =\sum_{v=1}^{n-1} \Delta_{v}\left(\hat{a}_{n v}\right) \lambda_{v} P_{v} s_{v}+\sum_{v=1}^{n-1} \hat{a}_{n, v+1} \Delta \lambda_{v} P_{v} s_{v} \\
& -\sum_{v=1}^{n-1} \hat{a}_{n, v+1} \lambda_{v+1} p_{v+1} s_{v}+a_{n n} \lambda_{n} P_{n} s_{n} \\
& =I_{n, 1}+I_{n, 2}+I_{n, 3}+I_{n, 4} .
\end{aligned}
$$

To complete the proof of Theorem 3 , it is sufficient to show that

$$
\sum_{n=1}^{\infty}\left(P_{n} / p_{n}\right)^{\delta k+k-1}\left|I_{n, r}(x)\right|^{k}<\infty, \text { for } r=1,2,3,4
$$

\section{Proof of Theorem 4}

The convergence of the Fourier series at $t=x$ is a local property of $f$ (i.e., it depends only on the behaviour of $f$ in an arbitrarily small neighbourhood of $x$ ), and hence the summability of the Fourier series at $t=x$ by any regular linear summability method is also a local property of $f$. Since the behaviour of the Fourier series, as far as convergence is concerned, for a particular value of $x$ depends on the behaviour of the function in the immediate neighbourhood of this point only, hence the truth of Theorem 4 is a consequence of Theorem 3 and Lemma 5 (see [9]).

\section{Conclusions}

Corollary 1. If we take $\delta=0$ in Theorem 3, then we obtain Theorem 1 dealing with $\left|A, p_{n}\right|_{k}$ summability.

Corollary 2. If we take $a_{n v}=\frac{p_{v}}{P_{n}}$ in Theorem 3, then we obtain a new theorem concerning with $\left|\bar{N}, p_{n} ; \delta\right|_{k}$ summability factors of Fourier series.
Corollary 3. If we take $a_{n v}=\frac{p_{v}}{P_{n}}$ and $p_{n}=1$ for all values of $\mathrm{n}$ in Theorem 3, then we get a result concerning $|C, 1 ; \delta|_{k}$ summability factors of Fourier series (see [10]).

\section{Acknowledgments}

This work was supported by Research Fund of Ahi Evran University, Project Number: FEF.A4.16.004.

\section{References}

1. S. Baron, "Local property of Fourier series", Tartu Riikl. Ül. Toimetised, Vih., 1965, 177, 106-120.

2. S. N. Bhatt, "An aspect of local property of summability of the factored Fourier series", Proc. Natl. Inst. Sci. India, 1960, 26, 69-73.

3. H. Bor, "On two summability methods", Math. Proc. Cambridge Philos Soc., 1985, 97, 147-149.

4. H. Bor," On the absolute Riesz summability factors", Rocky Mountain J. Math., 1994, 24, 1263-1271.

5. H. Bor, "Local property of summability of factored Fourier series", Bull. Inst. Math. Acad. Sinica,1989, 17, 165-170.

6. H. Bor, "On the local property of summability of factored Fourier series", J. Math. Anal. Appl.,1992, $163,220-226$.

7. H. Bor, "On the local property of $\left|\bar{N}, p_{n} ; \delta\right|_{k}$ summability of factored Fourier series", J. Math. Anal. Appl., 1993, 179, 646-649.

8. H. Bor, "Local properties of Fourier series", Int. J. Math. Sci., 2000, 23, 703-709.

9. K. K. Chen, "Functions of bounded variation and the Cesaro means of Fourier series", Acad. Sin. Sci.,1945, Record 1, 283-289,

10. T. M. Flett, " On an extension of absolute summability and some theorems of Littlewood and Paley", Proc. London Math. Soc., 1957, 7, 113-141.

11. G. H. Hardy, "Divergent Series", Oxford Univ. Press, Oxford, 1949.

12. M. Izumi, S. Izumi, "Localization problem of the absolute Riesz and absolute Nörlund summabilities of Fourier series", Canad. J. Math., 1970, 22, 615-625.

13. K. Matsumoto, "Local property of the summability", Tôhoku Math. J., 1956, 2, 114-124.

14. S. M. Mazhar, "On the summability factors of infinite series", Publ. Math. Debrecen, 1966, 13,229-236.

15. K. N. Mishra, "Multipliers for summability of Fourier series", Bull. Inst. Math. Acad. Sinica,1986, 14, 431438.

16. H. S. Özarslan, "A note on $\left|\bar{N}, p_{n}\right|_{k}$ summability factors", Int. J. Pure Appl. Math.,2004, 13, 485-490. 
Proc. of the Fourth International Conference on Advances in Computing, Electronics and Communication - ACEC 2016.

Copyright (C) Institute of Research Engineers and Doctors. All rights reserved.

ISBN: 978-1-63248-113-9 doi: 10.15224/ 978-1-63248-113-9-07

17. H. S. Özarslan, H. N. Öğdük, "Generalizations of two theorems on absolute summability methods", Aust. J. Math. Anal. Appl., 2004, 1(2), Art. 13,7.

18. W. T. Sulaiman, "Inclusion theorems for absolute matrix summability methods of an infinite series (IV)", Indian J. Pure and Appl. Math., 2003, 34 (11), 5471557.

19. N. Tanovic-Miller, "On strong summability", Glasnik Matematicki, 1979, 34 (14), 87-97.

20. E. C. Titchmarsh, " The Theory of Functions", Oxford University Press London, 1961.

21. Ş. Yildız, "A new theorem on local properties of factored Fourier series", Bull Math. Anal. Appl., 2016, $8(2), 1-8$. 